\title{
The Joy of Nanoscale Imaging and Spectroscopy in a Low Accelerating Voltage Scanning Transmitted Electron Microscope
}

\author{
Hendrix Demers ${ }^{1}$, Nicolas Brodusch ${ }^{2}$, Raynald Gauvin ${ }^{2}$, Michel L. Trudeau ${ }^{1}$, and Karim Zaghib ${ }^{1}$ \\ 1. Centre d'excellence en électrification des transports et stockage d'énergie, Hydro-Québec, Varennes, \\ Québec, Canada. \\ 2. Department of Mining and Materials Engineering, McGill University, Montreal, Quebec, Canada.
}

For developing new technologies, it is important to characterize materials at the nanoscale. To achieve high resolution, a field emission scanning electron microscope (FE-SEM) with low voltage scanning transmitted electron microscope mode (STEM) was recently developed. The HITACHI SU-9000 provides low accelerating voltage, deceleration mode, and energy-filtering of the electron signals (secondary and backscattered electrons), low voltage STEM bight-field (BF) and dark-field (DF) imaging capability are possible on this instrument to allow the characterization of materials at the nanoscale with various types of contrasts. Furthermore, the instrument has diffraction analysis, electron energy loss spectrometry (EELS), and energy dispersive spectrometry (EDS) systems, which allow crystallographic and chemical characterization of materials nanostructures.

Low accelerating voltage $(30 \mathrm{kV})$ lattice imaging was demonstrated by Joy on a multi wall carbon nanotube in BF mode [1]. A later study has explained the method and gave other materials examples [2]. Figure 1 shows a lattice image of a rutile $\mathrm{TiO}_{2}$ specimen. The Fourier transform of the lattice fringes indicates a distance of $0.34 \mathrm{~nm}$ for the dark grain and $0.35 \mathrm{~nm}$ for the bright grain. The lattice is not present for all grains as the condition for lattice visibility depends on the defocus and the lattice spacing. For a specific defocus value, only a range of lattice spacing value is visible, which explain why the two grains have similar lattice spacing.

The high resolution imaging is complemented by two analytical systems: electron energy loss spectrometry (EELS) and energy dispersive spectrometry (EDS). Examples of low voltage EELS were previously presented to validate the possibility of low voltage EELS [3, 4]. This instrument have three sensors for energy-filtering imaging. Using the same conditions as used in Figure 1, an energy-filtered lattice image was obtained as shown in Figure 2. Energy filtering improves the contrast of the bright-field image. In Figure 2, the micrograph was formed using electrons with the energy loss of the surface plasmon (Fig.2A) and the volume plasmon (Fig.2B). The surface plasmon micrograph shows a bright contrast at the edge of the specimen while the volume plasmon micrograph shows a better contrast across the full range of thickness of the specimen. We are presently developing simultaneous acquisition of EELS and EDS spectra to correctly characterize the specimen composition by combining these two complementary techniques.

These examples show the high resolution imaging and analysis possibilities of low voltage STEM obtained with an instrument like the SU-9000 for characterizing materials at the nanoscale, while at the same time minimizing the electron probe damage by reducing the dose used. 


\section{References:}

[1] V. Van Ngo et al, Microscopy Today 15 (2007) pp. 12-16.

[2] M. Konno et al, Ultramicroscopy 145 (2014) pp. 28-35.

[3] T. Sunaoshi et al, Microscopy and Microanalysis 22 (2016), pp. 604-605.

[4] Y. Yamazawa et al, Microscopy and Microanalysis 22 (2016), pp. 50-51.
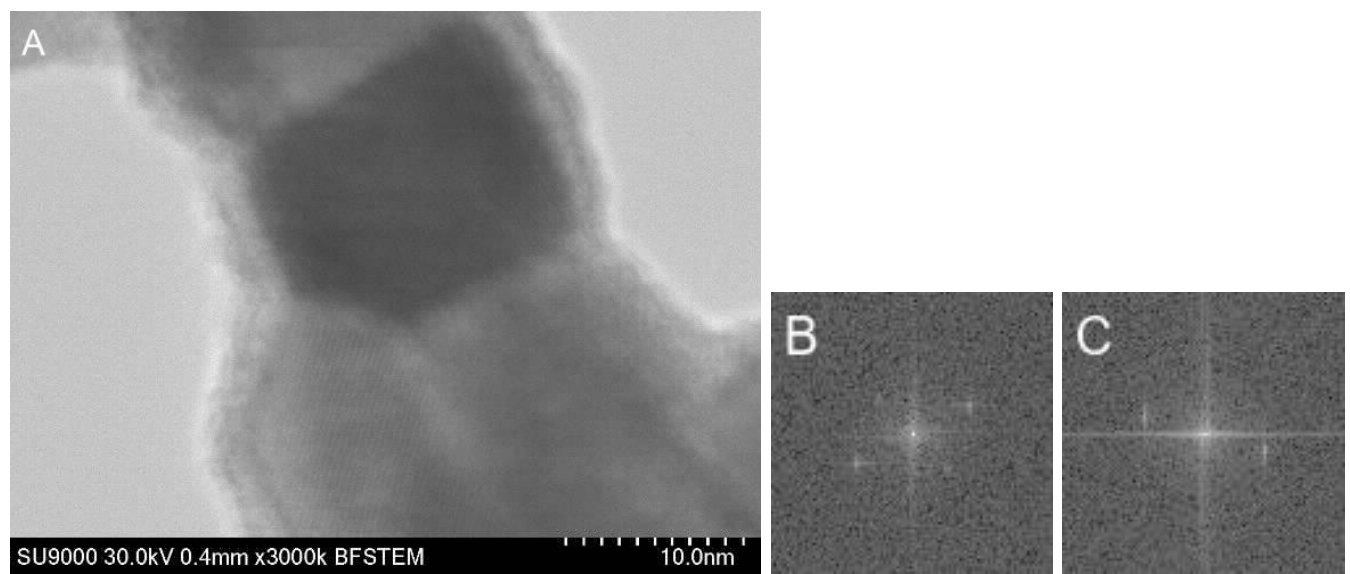

Figure 1. Example of lattice imaging of $\mathrm{TiO}_{2}$ (rutile). A Bright-field micrograph. B FT of the lattice fringes in the dark grain with a lattice spacing of $0.34 \mathrm{~nm}$. C FT of the lattice fringes in the bright grain with a lattice spacing of $0.35 \mathrm{~nm}$.
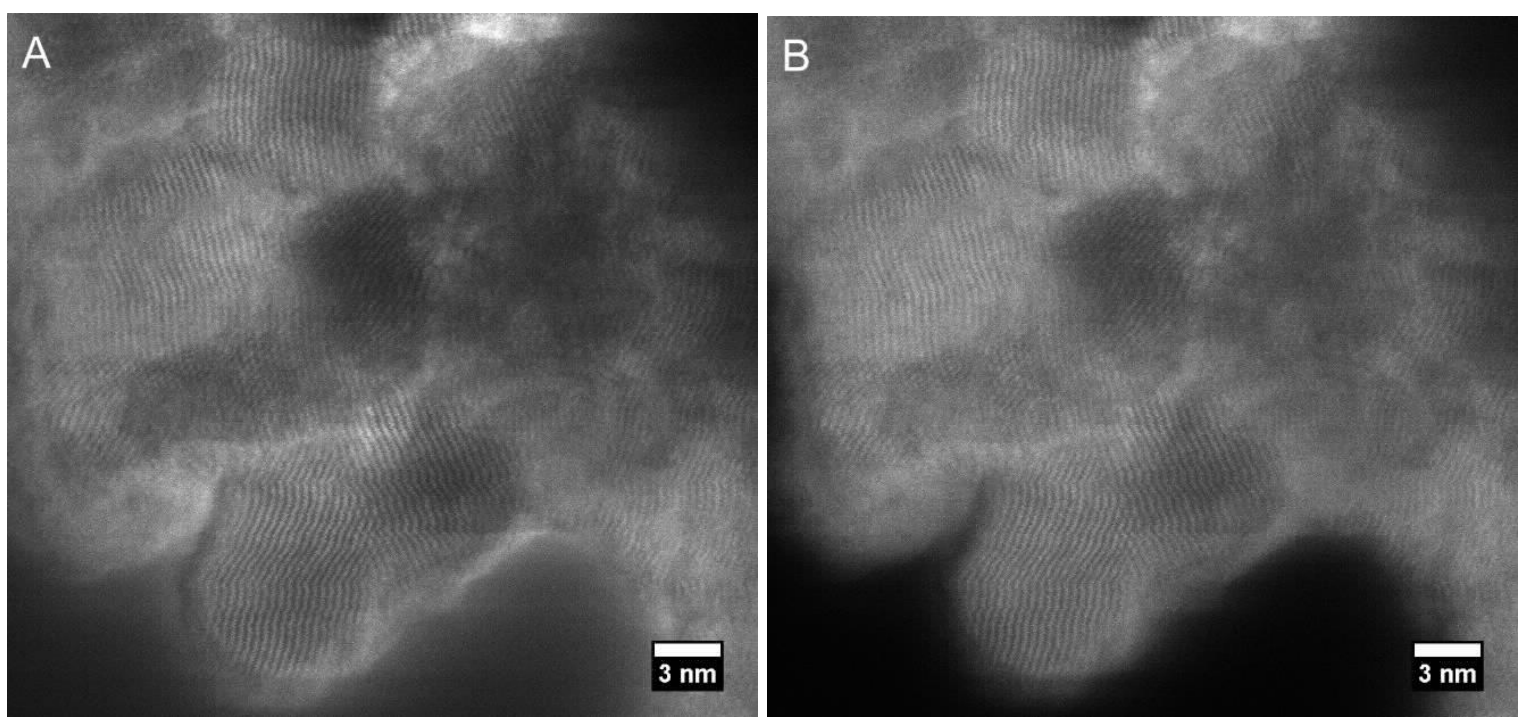

Figure 2. Example of lattice imaging of $\mathrm{TiO}_{2}$ using electron energy loss spectrometry (EELS) signal. A EFTEM micrograph using the surface plasmon region. B EFTEM micrograph using the volume plasmon region. 